\title{
Well-Posedness of MultiCriteria Network Equilibrium Problem
}

\author{
W. Y. Zhang \\ The School of Economic Mathematics, Southwestern University of Finance and Economics, Chengdu, \\ 610074, China \\ Correspondence should be addressed to W. Y. Zhang; zhangwy@swufe.edu.cn
}

Received 19 October 2013; Accepted 6 January 2014; Published 20 February 2014

Academic Editor: Bashir Ahmad

Copyright @ 2014 W. Y. Zhang. This is an open access article distributed under the Creative Commons Attribution License, which permits unrestricted use, distribution, and reproduction in any medium, provided the original work is properly cited.

New notions of $\epsilon$-equilibrium flow and $\xi_{k_{0}}$ - $\epsilon$-equilibrium flow of multicriteria network equilibrium problem are introduced; an equivalent relation between vector $\epsilon$-equilibrium pattern flow and $\xi_{k_{0}}-\epsilon$-equilibrium flow is established. Then, the well-posedness of multicriteria network equilibrium problem is discussed.

\section{Introduction}

For a long time, real-valued functions have played a central role in network equilibrium problems. Recently, motivated by applications to real-world situations, much attention has been attracted to multicriteria network equilibrium problems, that is, equilibrium problems with vector-valued cost functions. Different concepts of vector equilibrium flow have been introduced and the existence of such flows has been investigated by various authors (refer, first, to [1], in which Chen and Yen generalized Wardrop's scalar equilibrium principle to vector equilibrium principle, which asserts that users only choose Pareto optimal or efficient routes to travel on, and, among the others, to [2-6]).

Traffic equilibrium problem always depends on some parameters, because people's intuitive judgment plays a central role in route choices and it is impossible to have a precise estimation of the trip cost available to many paths. Therefore, some of the factors involved in transportation networks may be regarded as perturbing parameters. We were motivated to study the behavior of perturbations of multicriteria network equilibrium problems and cope with well-posed issues in the framework of traffic equilibrium problems. The notion of well-posedness, which can be useful for numerical purposes, is based on the behavior of either minimizing or maximizing sequences (Tychonov well-posedness). These notions for scalar optimization have been extensively investigated in many papers (cf., e.g., Dontchev and Zolezzi [7] in which a good list of basic references can be found). In the last decades, some extensions of this concept for vector optimization problems appeared; see [8-10] and the references therein. In the paper, we introduce the concept of well-posedness of multicriteria network equilibrium problems. As such, we obtain a sufficient condition that multicriteria network equilibrium problems are well-posed.

We now outline the remainder of the paper. Section 2 is devoted to the detailed description of the traffic network model. In Section 3, we introduce new notions of parametric equilibrium flows and obtain an equivalent relation between $\epsilon$-equilibrium pattern flow and $\xi_{k_{0}}-\epsilon$-equilibrium flow. Finally, we discuss the well-posedness of multicriteria network equilibrium problem.

\section{Preliminaries}

Consider a traffic network $[I, L]$, where $I$ denotes the set of origin and destination $(\mathrm{O} / \mathrm{D})$ pairs and $L$ is the set of directed arcs. We will suppose that $I$ has $l$ members. Let $i=(x, y) \in I$, which connects origin point $x$ with destination point $y$. Then a sequence

$$
\left(x, x_{1}\right),\left(x_{1}, x_{2}\right), \ldots,\left(x_{k-1}, y\right)
$$

of contiguous links in $L$ is called a path (or chain) from $x$ to $y$. Denote by $k$ the set of routes from $x$ to $y$ which traverse no link twice. Let $K_{i}$ be the set of paths that connect an O/D pair $i \in I$. Then $K_{i}$ is a finite set and

$$
K=\bigcup_{i \in I} K_{i}
$$

is a finite set too. We will suppose that $K$ has $m$ members. 
For a given path $k \in P_{i}$, let $v_{k}$ denote the traffic flow on this path and let $v=\left(v_{1}, v_{2}, \ldots, v_{m}\right) \in R^{m}$ be a flow of network. We will assume, for the rest of this paper, that the demand of network is fixed for each O/D pair; that is, $\sum_{k \in K_{i}} v_{k}=d_{i}$, where $d_{i}$ is a given demand for each $\mathrm{O} / \mathrm{D}$ pair $i$. A flow $v \geq 0$ satisfying the demand is called a feasible flow. $D$ is clearly a convex and compact set in $R^{m}$. Let there be given a vector of demands $d=\left(d_{1}, d_{2}, \ldots, d_{l}\right)$.

We will consider that the network system maintains an expected amount of flow in every path. That is, for every O/D pair $i \in I$ and every path $k \in K_{i}$, an expected flow $m_{k}(v)>0$ is given. For every O/D pair $i \in I$ and every path $k \in K_{i}$, we assume that $v_{k} \geq m_{k}(v)$. Let $m(v)=\left(m_{k}(v): k \in K_{i}, i \in I\right)$. Suppose that the set-valued mapping $Q: D \rightarrow 2^{D}$ is defined as $Q(v)=\{u \in D: u \geq m(v)\}$.

Let $Y$ be a Hausdorff topological vector space ordered by a pointed, closed convex cone $S \subset Y$ with $k_{0}, e \in \operatorname{int} S$. We denote by " $\leq$ " the ordering induced by $S$; that is,

$$
\begin{gathered}
x \leq y \quad \text { iff } y-x \in S, \\
x<y \quad \text { iff } y-x \in \operatorname{int} S .
\end{gathered}
$$

For every $i \in I$ and $k \in K_{i}$, we define the cost function of the path $k$ as a vector-valued function $C_{k}(v): R^{m} \rightarrow Y$; the mapping $C(v)=\left(C_{k}(v): k \in K_{i}, i \in I\right)$ is called the cost function of the network.

Definition 1 (see [11]). A vector $v$ is said to be an equilibrium pattern flow with a vector-valued cost function if and only if $v \geq m(v)$ and

$$
C_{r}(v)-C_{k}(v) \in S \backslash\{0\} \Longrightarrow\left(v_{r}-m_{r}(v)\right) e \notin \operatorname{int} S,
$$

for each $i \in I$ and any $k, r \in K_{i}$.

Remark 2. Notice the fact that $e \in \operatorname{int} S$ and $v_{r} \geq m_{r}(v)$ and the relationship $\left(v_{r}-m_{r}(v)\right) e \notin \operatorname{int} S$ in (4) actually is equivalent to $v_{r}-m_{r}(v)=0$. Therefore, (4) is equivalent to

$$
C_{r}(v)-C_{k}(v) \in S \backslash\{0\} \Longrightarrow v_{r}-m_{r}(v)=0 .
$$

Lemma 3 (see [11]). A vector flow $v \in D$ is an equilibrium pattern flow with a unilateral constraint if and only if $v$ is a solution to the quasivariational inequality: to find $v \in Q(v)$ such that

$$
\left\langle c(v),(u-v)^{T}\right\rangle \geq 0, \quad \forall u \in Q(v) .
$$

We now introduce a concept of approximate equilibria for the family of network equilibrium problems. Let $v \in D$ be a flow of the network $[I, L]$, and let $\epsilon \geq 0$. For each $i \in I$, denote

$$
\begin{aligned}
& G_{i, \epsilon}(v) \\
& =\left\{r \in K_{i}:\left(C_{r}(v)-\epsilon k_{0}-S \backslash\{0\}\right) \cap\left\{C_{k}(v), k \in K_{i}\right\}=\emptyset\right\} .
\end{aligned}
$$

Definition 4. A vector $v$ is said to be an $\epsilon$-equilibrium pattern flow with a vector-valued cost function if and only if $v \geq m(v)$ and

$$
v_{t} \leq m_{t}(v)+\epsilon, \quad \forall t \in K_{i} \backslash G_{i, \epsilon}(v)
$$

Remark 5. When $\epsilon=0$, the $\epsilon$-equilibrium pattern flow reduces to the equilibrium pattern flow as shown in Definition 1 . Namely, a vector $v \in D$ is a 0 -equilibrium pattern flow if and only if $v \in D$ is an equilibrium pattern flow.

In fact, assume that $v \in Q(v)$ is a 0 -equilibrium pattern flow but not an equilibrium pattern flow. Then, there exist $i \in I$ and $k, r \in K_{i}$ satisfying $C_{r}(v)-C_{k}(v) \in S \backslash\{0\}$ and $v_{r}>m_{r}(v)$. However, by Definition 4 and $v \in D$ being a 0 equilibrium pattern flow, $r \in G_{i, 0}(v)$. Hence, we have, for all $k \in K_{i}, C_{k}(v) \notin C_{r}(v)-S \backslash\{0\}$, which is a contradiction. Conversely, assume that $v \in Q(v)$ is an equilibrium pattern flow but not a 0 -equilibrium pattern flow. Then, there exists $r \in K_{i} \backslash G_{i, 0}(v)$ with $v_{r}>m_{r}(v)$. From the definition of $G_{i, 0}(v)$, there exists $k \in K_{i}$ satisfying $C_{k}(v) \in C_{r}(v)-S \backslash\{0\}$. From (5), $v_{r}=m_{r}(v)$. This is a contradiction.

\section{Well-Posedness of Multicriteria Network Equilibrium Problem}

The following real-valued function is of fundamental importance to our current analysis. The original version is due to what Gerstewitz (Tammer) [12] published in German.

Definition 6. Let $k_{0} \in \operatorname{int} S$ and $E \subset Y$. Gerstewitz's function $\xi_{k_{0}}: E \rightarrow R$ is defined by

$$
\xi_{k_{0}}(y)=\min \left\{t \in R \mid y \in t k_{0}-S\right\}, \quad \forall y \in E .
$$

By Theorem 2.1 of [13] and Lemmas 3 and 4 of [14], we have the following results.

Lemma 7. Let $k_{0} \in$ int $S$ and $E \subset Y$. For each $l \in R$ and $y \in E$, we have the following results:

(i) $\xi_{k_{0}}(y)<l \Leftrightarrow y \in l k_{0}-\operatorname{int} S$;

(ii) $\xi_{k_{0}}(y) \leq l \Leftrightarrow y \in l k_{0}-S$;

(iii) $\xi_{k_{0}}(y) \geq l \Leftrightarrow y \notin l k_{0}-\operatorname{int} S$;

(iv) $\xi_{k_{0}}(y)>l \Leftrightarrow y \notin l k_{0}-S$;

(v) $\xi_{k_{0}}(\cdot)$ is a continuous and strictly monotone function; namely,

$\xi_{k_{0}}\left(y_{1}\right)>\xi_{k_{0}}\left(y_{2}\right), \quad$ if $y_{1}, y_{2} \in E, y_{1}-y_{2} \in \operatorname{int} S$

(vi) $\xi_{k_{0}}(\cdot)$ is subadditive; namely,

$$
\xi_{k_{0}}\left(y_{1}+y_{2}\right) \leq \xi_{k_{0}}\left(y_{1}\right)+\xi_{k_{0}}\left(y_{2}\right), \quad \forall y_{1}, y_{2} \in E
$$

(vii) $\xi_{k_{0}}\left(l k_{0}\right)=l$, for all $l \in R$;

(viii) $\xi_{k_{0}}\left(y+l k_{0}\right)=l+\xi_{k_{0}}(y)$, for all $l \in R, y \in E$.

Definition 8 (see [11]). A vector $v$ is said to be a $\xi_{k_{0}}$ equilibrium pattern flow if and only if $v \geq m(v)$ and

$$
\xi_{k_{0}}\left(C_{r}(v)\right)-\xi_{k_{0}}\left(C_{k}(v)\right)>0 \Longrightarrow v_{r}-m_{r}(v)=0,
$$

for each $i \in I$ and $k, r \in K_{i}$. 
Abstract and Applied Analysis

3

Now let $v \in D$ be a flow of network $[I, L]$, and let $\epsilon \geq 0$. For each $i \in I$, denote

$$
\begin{gathered}
g_{i}^{\prime}(v)=\min \left\{\xi_{k_{0}}\left(C_{k}(v)\right), k \in K_{i}\right\}, \\
G_{i, \epsilon}^{\prime}(v)=\left\{r \in K_{i}: \xi_{k_{0}}\left(C_{r}(v)\right) \leq g_{i}^{\prime}(v)+\epsilon\right\} \subset K_{i} .
\end{gathered}
$$

Definition 9. Let $\epsilon \geq 0$. A vector $v$ is said to be a $\xi_{k_{0}}-\epsilon$ equilibrium pattern flow if and only if $v \geq m(v)$ and

$$
v_{t} \leq m_{t}(v)+\epsilon, \quad \forall t \in K_{i} \backslash G_{i, \epsilon}^{\prime}(v) .
$$

Theorem 10. If $v^{n} \in Q\left(v^{n}\right)$ is a $\xi_{k_{0}}-\epsilon_{n}$-equilibrium pattern flow, $v^{n} \rightarrow v$, and $m, C$ are continuous mappings, then $v$ is a $\xi_{k_{0}}$-equilibrium pattern flow.

Proof. Let a sequence $v^{n} \in D$ be a $\xi_{k_{0}}-\epsilon_{n}$-equilibrium pattern flow, let $v^{n} \rightarrow v$, and let $u \in Q(v)$ be arbitrarily chosen. From the continuity of $m$, compactness of $D$, and $v^{n} \in Q\left(v^{n}\right)$, we have $v \in Q(v)$. Moreover, it follows from the continuity of $m$ and the compactness of $D$ that, for every $u \in Q(v)$, there exists a sequence $\left\{u^{n}: u^{n} \geq m\left(v^{n}\right)\right\}$ satisfying $u^{n} \rightarrow u$.

Let $i \in I$ and $\epsilon_{n} \geq 0$; define

$$
\begin{gathered}
g_{i}\left(v^{n}\right)=\min \left\{\xi_{k_{0}} \circ C_{k}\left(v^{n}\right), k \in K_{i}\right\}, \\
G_{i}\left(v^{n}\right):=G_{i, \epsilon_{n}}^{\prime}\left(v^{n}\right)=\left\{t \in K_{i}: \xi_{k_{0}} \circ C_{t}\left(v^{n}\right) \leq g_{i}\left(v^{n}\right)+\epsilon_{n}\right\}, \\
G_{i}^{1}\left(v^{n}\right)=\left\{t \in G_{i}\left(v^{n}\right), u_{t}^{n}>v_{t}^{n}\right\}, \\
G_{i}^{2}\left(v^{n}\right)=\left\{t \in G_{i}\left(v^{n}\right), u_{t}^{n} \leq v_{t}^{n}\right\} .
\end{gathered}
$$

Clearly, $G_{i}\left(v^{n}\right)=G_{i}^{1}\left(v^{n}\right) \cup G_{i}^{2}\left(v^{n}\right)$.

By Definition 9, we have

$$
v_{t}^{n} \leq m_{t}\left(v^{n}\right)+\epsilon_{n}, \quad \forall t \in K_{i} \backslash G_{i}\left(v^{n}\right) .
$$

For $v^{n} \in D$ and $u^{n} \in Q\left(v^{n}\right)$, we have

$$
\begin{gathered}
\left\langle\xi_{k_{0}} \circ C\left(v^{n}\right),\left(u^{n}-v^{n}\right)^{T}\right\rangle \\
=\sum_{i=1}^{l} \sum_{t \in K_{i}} \xi_{k_{0}} \circ C_{t}\left(v^{n}\right)\left(u_{t}^{n}-v_{t}^{n}\right) \\
=\sum_{i=1}^{l}\left[\sum_{t \in G_{i}^{1}\left(v^{n}\right)} \xi_{k_{0}} \circ C_{t}\left(v^{n}\right)\left(u_{t}^{n}-v_{t}^{n}\right)\right. \\
+\sum_{t \in G_{i}^{2}\left(v^{n}\right)} \xi_{k_{0}} \circ C_{t}\left(v^{n}\right)\left(u_{t}^{n}-v_{t}^{n}\right) \\
\left.+\sum_{t \in K_{i} \backslash G_{i}\left(v^{n}\right)} \xi_{k_{0}} \circ C_{t}\left(v^{n}\right)\left(u_{t}^{n}-v_{t}^{n}\right)\right]
\end{gathered}
$$

$$
\begin{aligned}
\geq \sum_{i=1}^{l} & {\left[\sum_{t \in G_{i}^{1}\left(v^{n}\right)} g_{i}\left(v^{n}\right)\left(u_{t}^{n}-v_{t}^{n}\right)\right.} \\
& +\sum_{t \in G_{i}^{2}\left(v^{n}\right)}\left(g_{i}\left(v^{n}\right)+\epsilon_{n}\right)\left(u_{t}^{n}-v_{t}^{n}\right)
\end{aligned}
$$$$
\left.+\sum_{t \in K_{i} \backslash G_{i}\left(v^{n}\right)} \xi_{k_{0}} \circ C_{t}\left(v^{n}\right)\left(u_{t}^{n}-m_{t}\left(v^{n}\right)-\epsilon_{n}\right)\right]
$$

$$
\begin{aligned}
& \geq \sum_{i=1}^{l}[ \sum_{t \in G_{i}^{1}\left(v^{n}\right)} g_{i}\left(v^{n}\right)\left(u_{t}^{n}-v_{t}^{n}\right) \\
&+\sum_{t \in G_{i}^{2}\left(v^{n}\right)}\left(g_{i}\left(v^{n}\right)+\epsilon_{n}\right)\left(u_{t}^{n}-v_{t}^{n}\right) \\
&+\sum_{t \in K_{i} \backslash G_{i}\left(v^{n}\right)} g_{i}\left(v^{n}\right)\left(u_{t}^{n}-m_{t}\left(v^{n}\right)\right) \\
&\left.-\sum_{t \in K_{i} \backslash G_{i}\left(v^{n}\right)} \epsilon_{n} \xi_{k_{0}} \circ C_{t}\left(v^{n}\right)\right] \\
&=\sum_{i=1}^{l}\left[\sum_{t \in K_{i}} g_{i}\left(v^{n}\right) u_{t}^{n}-\sum_{t \in G_{i}\left(v^{n}\right)} g_{i}\left(v^{n}\right) v_{t}^{n}\right. \\
& \quad-\sum_{t \in K_{i} \backslash G_{i}\left(v^{n}\right)} g_{i}\left(v^{n}\right) m_{t}\left(v^{n}\right)+\sum_{t \in G_{i}^{2}\left(v^{n}\right)} \epsilon_{n}\left(u_{t}^{n}-v_{t}^{n}\right) \\
&\left.\quad \sum_{t \in K_{i} \backslash G_{i}\left(v^{n}\right)} \epsilon_{n} \xi_{k_{0}} \circ C_{t}\left(v^{n}\right)\right]
\end{aligned}
$$

$$
\begin{aligned}
& =\sum_{i=1}^{l}\left[\sum_{t \in K_{i}} g_{i}\left(v^{n}\right) u_{t}^{n}-\sum_{t \in K_{i}} g_{i}\left(v^{n}\right) v_{t}^{n}\right. \\
& +\sum_{t \in K_{i} \backslash G_{i}\left(v^{n}\right)} g_{i}\left(v^{n}\right)\left(v_{t}^{n}-m_{t}\left(v^{n}\right)\right) \\
& \left.+\sum_{t \in G_{i}^{2}\left(v^{n}\right)} \epsilon_{n}\left(u_{t}^{n}-v_{t}^{n}\right)-\sum_{t \in K_{i} \backslash G_{i}\left(v^{n}\right)} \epsilon_{n} \xi_{k_{0}} \circ C_{t}\left(v^{n}\right)\right] \\
& =\sum_{i=1}^{l}\left[g_{i}\left(v^{n}\right)\left(\sum_{t \in K_{i}} u_{t}^{n}-\sum_{t \in K_{i}} v_{t}^{n}\right)\right. \\
& +\sum_{t \in K_{i} \backslash G_{i}\left(v^{n}\right)} g_{i}\left(v^{n}\right)\left(v_{t}^{n}-m_{t}\left(v^{n}\right)\right) \\
& \left.+\sum_{t \in G_{i}^{2}\left(v^{n}\right)} \epsilon_{n}\left(u_{t}^{n}-v_{t}^{n}\right)-\sum_{t \in K_{i} \backslash G_{i}\left(v^{n}\right)} \epsilon_{n} \xi_{k_{0}} \circ C_{t}\left(v^{n}\right)\right] .
\end{aligned}
$$


Since $u^{n} \rightarrow u$, then, for every $i, \sum_{t \in K_{i}} u_{t}^{n} \rightarrow \sum_{t \in K_{i}} u_{t}$ when $n \rightarrow+\infty$. By $v^{n}, u \in D, \sum_{t \in K_{i}} u_{t}=\sum_{t \in K_{i}} v_{t}^{n}=d_{i}$. Thus, for each $i$,

$$
\sum_{t \in K_{i}} u_{t}^{n}-\sum_{t \in K_{i}} v_{t}^{n}=\sum_{t \in K_{i}} u_{t}^{n}-d_{i} \longrightarrow 0
$$

Letting $n \rightarrow \infty$, we conclude that

$$
\left\langle\xi_{k_{0}} \circ C(v),(u-v)^{T}\right\rangle \geq 0 .
$$

Therefore, from Lemma 3 (take $\xi_{k_{0}} \circ C=c$ ), $v$ is a $\xi_{k_{0}}{ }^{-}$ equilibrium pattern flow.

Set $C_{t}: R^{m} \rightarrow Y$ in the following form:

$$
C_{t}(v)=f_{t}(v) k_{0}, \quad \forall t \in K_{i}, i \in I,
$$

where $f_{t}(v): R^{m} \rightarrow R$.

Theorem 11. Let $\epsilon \geq 0$ and $C_{t}$ be defined as (20) for all $t \in K_{i}$, $i \in I . v$ is an $\epsilon$-equilibrium pattern flow with a vector-valued cost function if and only if $v$ is a $\xi_{k_{0}}-\epsilon$-equilibrium pattern flow.

Proof. Assume that $v$ is an $\epsilon$-equilibrium pattern flow but not a $\xi_{k_{0}}-\epsilon$-equilibrium pattern flow. Then, there exist an $\bar{i} \in I$ and a pair of $\bar{k}, \bar{r} \in K_{\bar{i}}$ satisfying

$$
\begin{gathered}
\xi_{k_{0}}\left(C_{\bar{r}}(v)\right)-\xi_{k_{0}}\left(C_{\bar{k}}(v)\right)+\epsilon>0, \\
v_{\bar{r}}-m_{\bar{r}}(v)>\epsilon .
\end{gathered}
$$

From $\xi_{k_{0}}\left(C_{\bar{r}}(v)\right)-\xi_{k_{0}}\left(C_{\bar{k}}(v)\right)-\epsilon>0$, we have

$$
\begin{aligned}
\xi_{k_{0}}\left(C_{\bar{k}}(v)-C_{\bar{r}}(v)+\epsilon k_{0}\right) \\
\quad=f_{\bar{k}}(v)-f_{\bar{r}}(v)+\epsilon \\
=\xi_{k_{0}}\left(f_{\bar{k}}(v) k_{0}\right)-\xi_{k_{0}}\left(f_{\bar{r}}(v) k_{0}\right)+\epsilon \\
=\xi_{k_{0}}\left(C_{\bar{k}}(v)\right)-\xi_{k_{0}}\left(C_{\bar{r}}(v)\right)+\epsilon<0 .
\end{aligned}
$$

By Lemma 7,

$$
C_{\bar{k}}(v)-C_{\bar{r}}(v)+\epsilon k_{0} \in-\operatorname{int} S \subset-S \backslash\{0\} .
$$

Thus,

$$
C_{\bar{k}}(v) \in C_{\bar{r}}(v)-\epsilon k_{0}-S \backslash\{0\} .
$$

This implies that $\bar{r} \in K_{\bar{i}} \backslash G_{\bar{i}, \epsilon}^{\prime}(v)$. It follows from $v$ being an $\epsilon$-equilibrium pattern flow that

$$
v_{\bar{r}}-m_{\bar{r}}(v) \leq \epsilon,
$$

which contradicts (22).

Conversely, assume that $v$ is a $\xi_{k_{0}}-\epsilon$-equilibrium pattern flow. Then, for any $i \in I$ and every path $r \in K_{i} \backslash G_{i, \epsilon}(v)$, we want to deduce $v_{r}-m_{r}(v) \leq \epsilon$.

It follows from $r \in K_{i} \backslash G_{i, \epsilon}(v)$ that there exists $k \in K_{i}$ satisfying

$$
C_{r}(v)-C_{k}(v)-\epsilon k_{0} \in S \backslash\{0\}
$$

Then,

$$
\xi_{k_{0}}\left(C_{r}(v)-C_{k}(v)-\epsilon k_{0}\right)=f_{r}(v)-f_{k}(v)-\epsilon \geq 0 .
$$

Since $C_{r}(v)-C_{k}(v)-\epsilon k_{0} \neq 0$, we have $f_{r}(v)-f_{k}(v)-\epsilon \neq 0$. Therefore,

$$
\begin{aligned}
\xi_{k_{0}}\left(C_{r}(v)\right)-\xi_{k_{0}}\left(C_{k}(v)\right)-\epsilon \\
\quad=\xi_{k_{0}}\left(C_{r}(v)-C_{k}(v)-\epsilon k_{0}\right)>0 .
\end{aligned}
$$

Hence, $r \in K_{i} \backslash G_{i, \epsilon}^{\prime}(v)$. By Definition 9 , we have $v_{r}-m_{r}(v) \leq$ $\epsilon$.

Definition 12. Let $\left\{\epsilon_{n}: \epsilon_{n} \geq 0, \epsilon_{n} \rightarrow 0\right\}$ be a sequence, let $v^{n} \in$ $D$ be an $\epsilon_{n}$-equilibrium flow for each $n$, and let $v^{n} \rightarrow v$; the network $[I, L]$ is called well-posed if $v$ is a traffic equilibrium flow.

Theorem 13. Let $C_{t}$ be defined as (20). If $m$ and $f_{t}$ are continuous mappings, then the network $[I, L]$ is well-posed.

Proof. Let $v^{n} \in D$ be an $\epsilon_{n}$-equilibrium pattern flow. From Theorem 11, $v^{n}$ is a $\xi_{k_{0}}-\epsilon_{n}$-equilibrium pattern flow. From Theorem 10 and $v^{n} \rightarrow v, v$ is a $\xi_{k_{0}}$-equilibrium pattern flow. By Theorem 11 (take $\epsilon=0$ ), $v \in D$ is an equilibrium pattern flow for the vector network equilibrium problem.

\section{Conflict of Interests}

The author declares that there is no conflict of interests regarding the publication of this paper.

\section{Acknowledgment}

This research was partially supported by the National Natural Science Foundation of China (Grant no. 11226231).

\section{References}

[1] G. Y. Chen and N. D. Yen, "On the variational inequality model for network equilibrium," Internal Report 196, Department of Mathematics, University of Pisa, 1993.

[2] P. Q. Khanh and L. M. Luu, "On the existence of solutions to vector quasivariational inequalities and quasicomplementarity problems with applications break to traffic network equilibria," Journal of Optimization Theory and Applications, vol. 123, no. 3, pp. 533-548, 2004.

[3] C. J. Goh and X. Q. Yang, "Vector equilibrium problem and vector optimization," European Journal of Operational Research, vol. 116, no. 3, pp. 615-628, 1999.

[4] S. J. Li and G. Y. Chen, "On relations between multiclass, multicriteria traffic network equilibrium models and vector variational inequalities," Journal of Systems Science and Systems Engineering, vol. 15, no. 3, pp. 284-297, 2006.

[5] S. J. Li, K. L. Teo, and X. Q. Yang, "A remark on a standard and linear vector network equilibrium problem with capacity constraints," European Journal of Operational Research, vol. 184, no. 1, pp. 13-23, 2008.

[6] X. Q. Yang and C. J. Goh, "On vector variational inequalities: application to vector equilibria," Journal of Optimization Theory and Applications, vol. 95, no. 2, pp. 431-443, 1997. 
[7] A. Dontchev and T. Zolezzi, Well-Posed Optimization Problems, vol. 1543 of Lecture Notes in Mathematics, Springer, Berlin, Germany, 1993.

[8] E. M. Bednarczuk, "An approach to well-posedness in vector optimization problems: consequences to stability," Control and Cybernetics, vol. 23, pp. 107-122, 1994.

[9] X. X. Huang, "Extended well-posedness properties of vector optimization problems," Journal of Optimization Theory and Applications, vol. 106, no. 1, pp. 165-182, 2000.

[10] E. Miglierina, E. Molho, and M. Rocca, "Well-posedness and scalarization in vector optimization," Journal of Optimization Theory and Applications, vol. 126, no. 2, pp. 391-409, 2005.

[11] Y. N. Wu, G. Y. Chen, and T. C. E. Cheng, "A vector network equilibrium problem with a unilateral constraint," Journal of Industrial and Management Optimization, vol. 6, no. 3, pp. 453464, 2010.

[12] C. Gerstewitz (Tammer), "Nichtkonvexe dualität in der vektoroptimierung," Wissenschaftliche Zeitschrift TH LeunaMerseburg, vol. 25, pp. 357-364, 1983.

[13] C. Gerth and P. Weidner, "Nonconvex separation theorems and some applications in vector optimization," Journal of Optimization Theory and Applications, vol. 67, no. 2, pp. 297320, 1990.

[14] S. J. Li, X. Q. Yang, and G. Y. Chen, "Vector Ekeland variational principle," in Vector Variational Inequalities and Vector Equilibria, F. Giannessi, Ed., pp. 321-333, Kluwer Academic, 2000. 


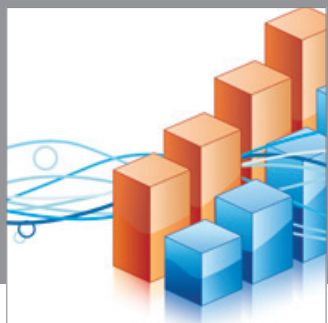

Advances in

Operations Research

mansans

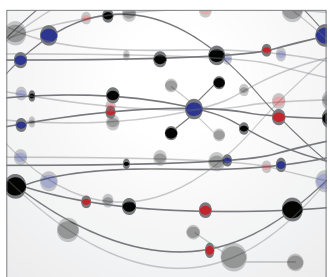

The Scientific World Journal
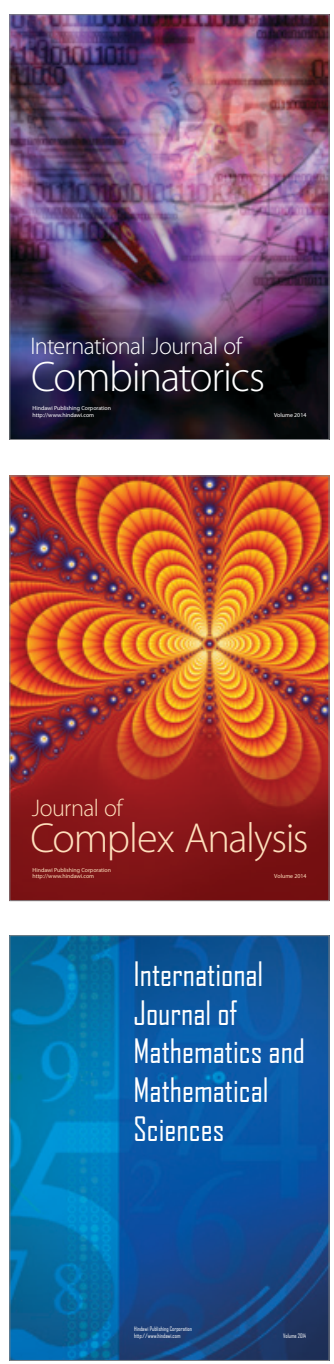
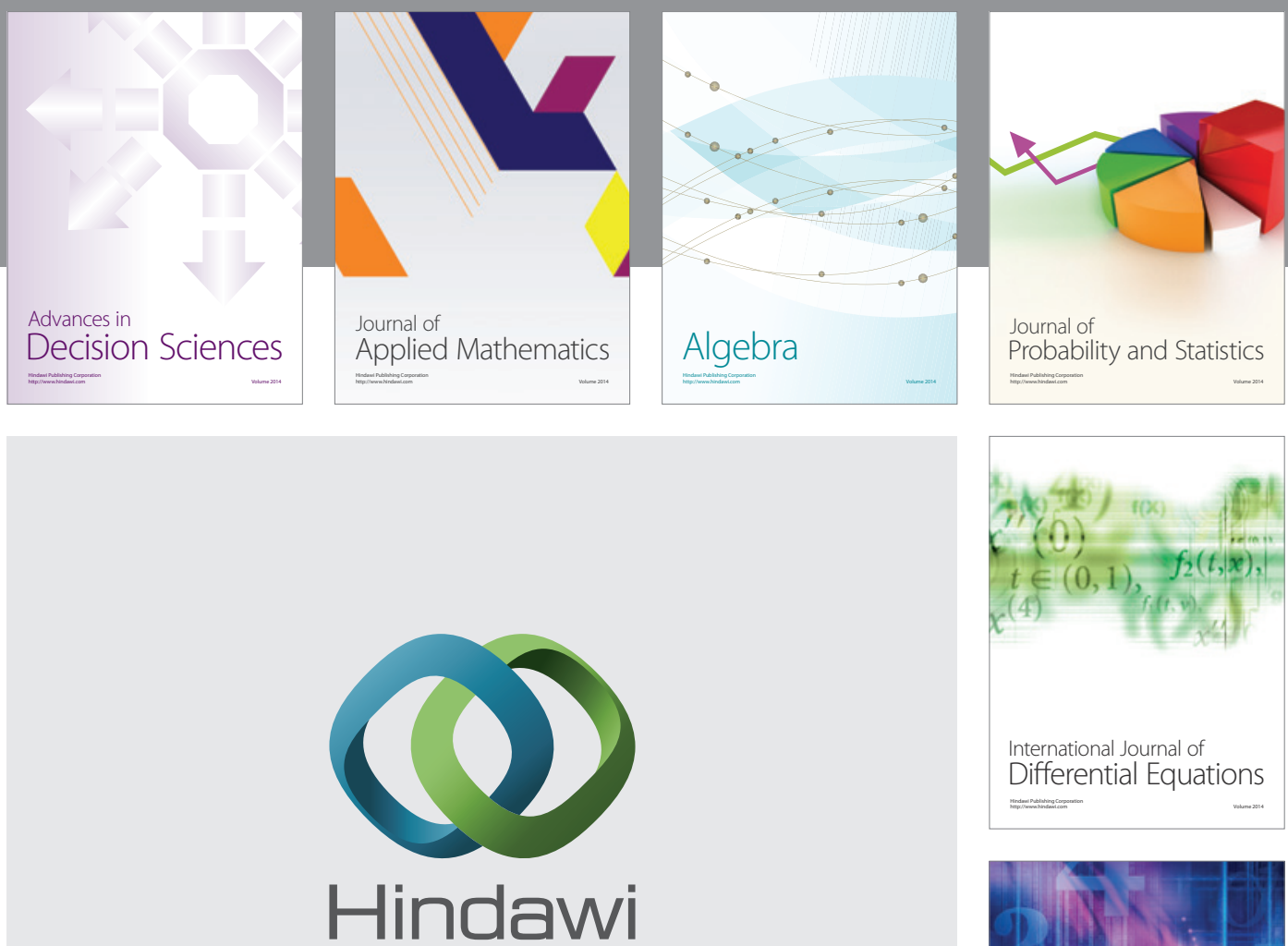

Submit your manuscripts at http://www.hindawi.com
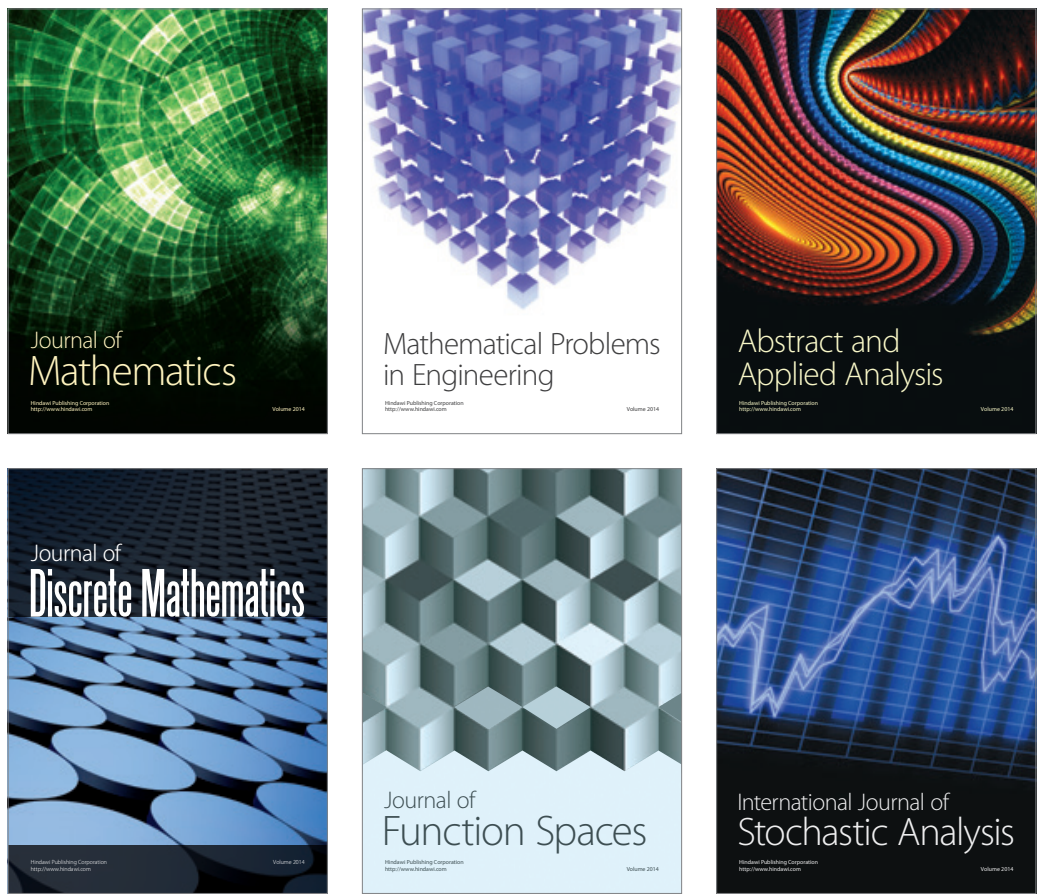

Journal of

Function Spaces

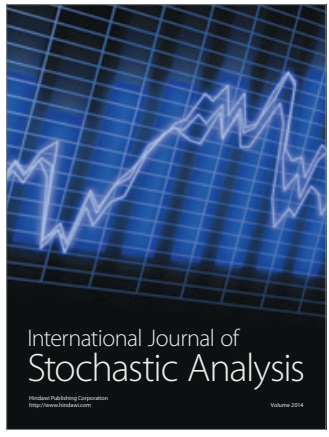

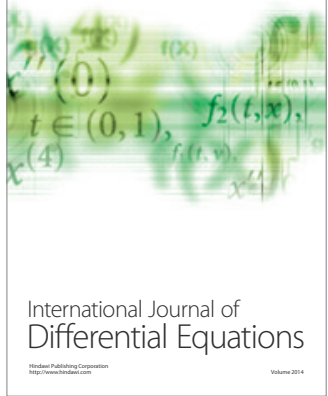
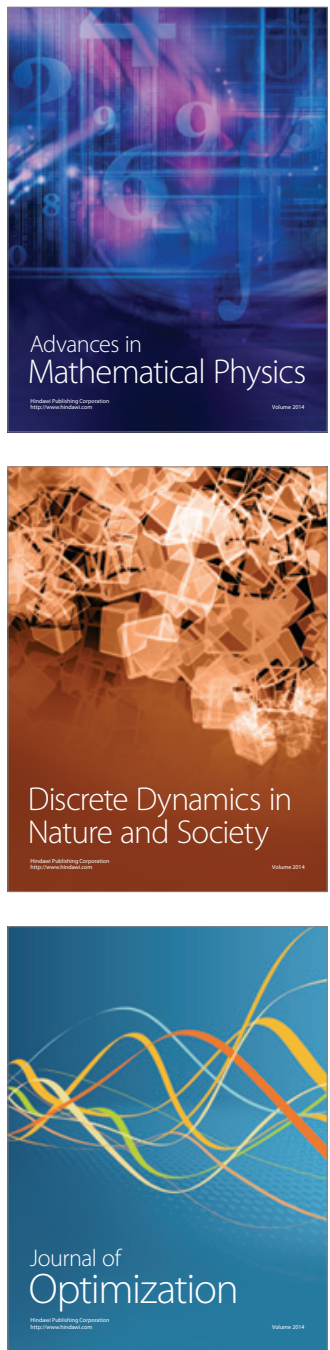